DOI: 110.2478/ausp-2018-0016

\title{
An Analysis of Ethnic Influence on Language: Mandarin or Xinjiang Mandarin?
}

\author{
Maomao GAO \\ Eötvös Loránd University (Budapest, Hungary) \\ Department of Intercultural Linguistics \\ maomaogao3707@gmail.com
}

\begin{abstract}
This paper aims to investigate lexical borrowings from ethnic languages to standard Mandarin. Data are collected through daily observation after years of living in Xinjiang, China. The data suggest that phonetic loans and hybrid loans are the major approaches in lexical borrowings from Uyghur, Russian, and Persian. Two motivations behind Uyghur borrowings into Mandarin are discussed: cultural borrowings and core borrowings. Cultural borrowings are new objects, concepts of ethnic origins, which are new to Han Chinese communities. Core borrowings are Mandarin words which have been replaced by Uyghur. However, core borrowings are not associated with the prestige of the donor language in this case; instead, frequency, marker, friendliness, and religious consideration are the major reasons. In addition, this paper analyses the strategies of lexical borrowings from ethnic languages in Mandarin. It further discusses the cultural backgrounds behind lexical borrowings. The lexical borrowings from ethnic languages into Mandarin suggest the openness and inclusiveness of Chinese language and culture.
\end{abstract}

Keywords: lexical borrowing, cultural borrowing, ethnic language, multiculturalism, Mandarin.

\section{Introduction}

Since the $17^{\text {th }}$ century, Han Chinese have been migrating to Xinjiang, a province with 15 ethnic minorities. Though the official languages are Mandarin and Uyghur, the Mandarin in this area has developed into a unique style under the ethnic influences. For example, the word for onion ${ }^{1}$ is 'yangcong' in standard Mandarin, while in Xinjiang Mandarin the word for onion has become 'piyazi', which is a word borrowed from Uyghur. Even though these two words refer to the

1 The translation of ethnic origin examples from Mandarin to English are my own throughout the article. 
same object, the use of 'piyazi' can only be found in this region. As a multi-ethnic region, the direct contact of various ethnic groups has contributed to a unique style of Mandarin spoken in Xinjiang.

There have been various studies on lexical borrowings of Mandarin from English, yet little research has focused on lexical borrowing from ethnic languages in Mandarin. This paper aims to explore the ethnic influences in Mandarin spoken in Xinjiang. It further presents the motivations behind lexical borrowings from ethnic languages in Xinjiang and the significance of lexical borrowings due to emotional connection among different ethnic groups.

\section{Why there are lexical borrowings}

Thomason-Kaufman (1988) have defined borrowings starting from two different aspects. First of all, borrowing can be the result of language transfer from native speakers to non-native speakers. On the one hand, native speakers adopt elements of a language and incorporate them into the recipient language. Moreover, non-native speakers impose the characteristics of their native language onto the recipient language. However, in a more restricted sense, borrowings may solely refer to the integration of foreign elements and speakers' native language (Haspelmath 2009: 36). Tsvetkow-Dyer (2016) pointed out that linguistic borrowings occur when different language communities have contact with each other, which results in lexical, phonological, morphological, and syntactic changes in recipient languages.

Haspelmath has suggested four forms of borrowings: cultural borrowing, core borrowing, therapeutic borrowing, and adoption vs imposition. There are various ways to either make up words for new concepts or extend the semantic meaning of existing words. In the case of cultural borrowings, some ethnic groups combine borrowed words together with new concepts from other languages, and these words become better known than the words in their native language. Later, these better known words override the native language. However, this may not happen if the ethnic groups perceive their languages as markers of their ethnic identity, i.e. they resort to purism. For example, there is a tendency to avoid English borrowings in French, yet several other European languages are more welcoming to English borrowings. The issue with cultural borrowings represents the measurement of the speaker's attitude. Thus, cultural borrowings are more likely to occur if there is no significant resistance among speakers (Haspelmath 2009).

Core borrowing refers to the replacement of words that already exist. These language changes relate to the social prestige of the donor language because the way we speak not only gets the meaning across but also projects an impression of us on others. In some cases, even though there is a word in speakers' native 
language for a concept, they adopt the borrowed word to convey the meaning. Therapeutic borrowings occur when the original words are not available. For example, if there are two words similar in spelling or pronunciation but with different meanings, lexical borrowings could avoid confusion (Haspelmath 2009).

Adoption refers to speakers borrowing features of a dominant language into their native language, and the borrowings might range from lexicon to language structure. Imposition means that non-native speakers unconsciously retain their language features in the language they are shifting to. Phonology and syntax borrowings are the major motivations in imposition. In fact, all retained words serve a purpose. For example, non-native speakers retain their language features as markers of their identity (Haspelmath 2009).

In sum, the above mentioned lexical borrowing strategies can be found in many languages. Mandarin is one of the languages which is resistant to lexical borrowings coming from foreign languages. Yet, the Mandarin spoken in Xinjiang, China, has adopted several ethnic language features.

\subsection{The history of lexical borrowings in Chinese}

According to Dai-Dong (1999), there are 56 ethnic nationalities in China. Among them, Han people accounts for over ninety percent of the total population, with the other 55 ethnic minorities making up the remaining ten percent of the population. Hans speak Chinese, and 53 ethnic minorities speak more than 80 languages. Two other nationalities, Man and Hui, have already adopted Chinese as their native tongue. Chinese is a Sino-Tibetan language which consists of seven major dialect groups in China: Mandarin, Wu, Xiang, Gan, Min, Kejia, and Yue. Among them, Mandarin used to be a dialect in the northern part of China, which later became Standard Mandarin, while the other six are referred to as southern dialects (Miao 2005: 19).

Chinese has been an influential language to its neighbouring countries, but it has borrowed foreign words along history. The Silk Road connected China to the West in the Western Han (BC 206-AD 24) and Eastern Han (AD 25-220) dynasties. Chinese borrowed words from languages of ancient Persia. Later, in the Sui (AD 581-618) and Tang (AD 618-907) dynasties, Chinese borrowed Buddhist terms from Sanskrit. Christianity entered China during the Ming (AD 1368-1644) and Qing (AD 1616-1911) dynasties. Christian terms were then translated and borrowed into Chinese. China's defeat in the Sino-British War (1840-1842) was a turning point, from which Western works have been introduced to China. In the late $19^{\text {th }}$ and early $20^{\text {th }}$ century, Chinese borrowed from Japanese mainly the graphic form, rather than meaning. From 1949 to the 1960s, Russian words were the most common borrowings. From 1970 to present, various words from different languages have been borrowed by Chinese (Miao 2005: 22-28). 


\subsection{Lexical borrowing approaches in Chinese}

Miao (2005) has demonstrated four major approaches of foreign borrowings into Chinese: phonemic loans, semantic loans, graphic loans, and hybrids. Phonemic loans refer to foreign words which are rendered in Mandarin phonemically. In this way, the words maintain the same phonetic sounds in Mandarin, and the phonetic sounds are the only similarity between the foreign words and those rendered in Chinese. However, another type of foreign loans goes beyond phonetic similarities as the phonetic sounds trigger semantic associations. For example, 'Kendeji' (肯德基) is the Chinese version of 'KFC' (Kentucky Fried Chicken). The last phonetic sound of the Chinese version 'ji' is 'chicken' in Chinese. Thus, the rendered Chinese version not only keeps the phonetic features of the brand, but it also associates the brand with one of its ingredients: chicken. However, the brand did not adopt 'ji' (鸡) in its written form in Chinese; instead, they use 'ji' (基). Note that in Chinese the same phonetic sound may have several written characters.

Semantic loans focus on the meaning of the foreign words when changed into Mandarin. One method is translating each morpheme of the foreign words and combining them into one word. Another method takes a broader look at the meaning of the foreign words and invents new words in Mandarin.

Graphic loans are foreign written forms which are borrowed into Mandarin. For example, many Japanese written forms were borrowed into Chinese from the 1930s to 1980s, and many graphic loans from the Latin alphabet have also entered Chinese people's lives: for instance, "CD" (Compact Disk) and "ATM" (Automated Teller Machine) (Miao 2005: 29-33).

Hybrids are words which are adapted to Chinese using a combination of semantic features and phonetic features. There are many variations in hybrid borrowings of foreign words. In hybrids, phonetic and semantic features are separate and independent. For example, 'AIDS' is 'ai4-zi1-bing4' in Chinese. Here, 'ai4zi1' is the phonetic equivalent of 'AIDS' and 'bing4' (disease) is added as a semantic feature. Graphic + phonetic/semantic hybrids in written form are another type of hybrids. For example, 'X-Men' is adapted to 'X-zhanjing'. 'Zhanjing' is the semantic feature of the hybrids, which means 'soldier' (Miao 2005: 34-36).

\section{Lexical borrowings of Mandarin in Xinjiang}

Even though Mandarin is the official language, there are more than 80 different languages spoken by different ethnic groups. Extensive research has been carried out concerning lexical borrowings of foreign words, yet little research focuses on ethnic influences on Mandarin in Xinjiang, China. Xinjiang is a province located in the north-western part of China. There are 47 ethnic nationalities with two 
major language groups. One distinct group is that of ethnic language speakers. The other group is formed by speakers of Han Chinese, which consists of almost all seven major Chinese dialects. Since the independence of the People's Republic of China, many speakers of Han Chinese from all parts of China have moved to Xinjiang. In addition, many other ethnic groups have been living there for centuries. Among them, the Uyghur ethnicity is one of the dominant groups in this region. The unique culture and historical background have shaped the language style (Lu 2011).

When Han Chinese moved to Xinjiang, they also brought Mandarin. This was not standard Mandarin but a variation of Mandarin (Lu 2011). Because most of the Han immigrants came from the northern part of China, they spoke Mandarin with slight differences from region to region. The spoken Mandarin gained more ethnic features as there were frequent contacts between Han Mandarin speakers and ethnic groups. Though Mandarin and Uyghur are both official languages in this region, Xinjiang Mandarin is widely used among many Uyghur speakers as well (Baki 2012). Initially, a few ethnic words used in Mandarin have attracted my attention to investigate the lexical borrowings in Mandarin in Xinjiang.

\subsection{Words of Uyghur origin}

The data presented in this paper were collected from ethnographic sources. The collected words are not originated from Mandarin or other Chinese dialects. However, they are commonly used by Han Mandarin speakers as well as ethnic language speakers in Xinjiang. In this chapter, I would like to apply Miao's (2005) approach to analyse the strategies of lexical borrowings of Mandarin in Xinjiang. The motivations behind the lexical borrowings will be discussed as well.

Many words of non-Chinese origin have already become part of daily communication. These are words that have equivalent words in standard Mandarin. For example, 'onion' is called 'piyazi' (皮牙孜) instead of 'yangcong' (洋葱) in standard Mandarin. It comes from the Uyghur 'piyaz'. In standard Mandarin, 'young boy' is referred to as 'nanhai' (男孩). In Xinjiang, it has changed to 'balangzi' (巴郎子); the Uyghur form is 'bala'. There are more words that are exclusive in Xinjiang. 'Nang' (睘) is a baked bread specialty which comes from the Uyghur 'nan'. 'Badanmu' (巴旦木), in English 'almond', comes from 'badam' in Uyghur. A few words related to entertainment also have been rendered into Mandarin. 'Dawazi' (达瓦孜) means 'tight-rope walking', 'mukamu' (木卡姆) denotes 'traditional twelve-chapter Uyghur music', and 'maixilaipu' (麦西来普) refers to 'gathering with music and dancing'. The original forms are 'dawaz', 'muqam', and 'meshrep' (Baki 2012, Lu 2011).

Among the above examples, it is clear that most words of Uyghur origin have been rendered into Mandarin through phonetic loans. Some of them are cultural 
borrowings, some are core borrowings. For example, 'Badanmu' (巴旦木), 'nang' (壊), 'dawazi' (达瓦孜), 'mukamu' (木卡姆), and 'maixilaipu' (麦西来普) keep their phonetic features when changed into Mandarin. As cultural borrowings, they introduce new concepts or activities to Mandarin speakers.

'Piyazi' (皮牙孜) sounds similar to 'piyaz' in Uyghur. Both the Mandarin and Uyghur indicate the same object, yet people have adopted the Uyghur-origin form instead of the standard Mandarin form: 'yangcong' (洋葱). Words like 'piyazi' ('onion') and 'balangzi' ('young boy') have been integrated into daily communication among different ethnic groups.

According to Haspelmath (2009), core borrowings are motivated by social prestige. However, I do not consider that these two borrowings were motivated by the social prestige of the donor language. Instead, I think three reasons may have influenced the borrowings.

Firstly, the core borrowing words are frequently used in daily communication among different ethnic groups. 'Piyazi' ('onion' and 'balangzi' ('young boy') are words that may occur in conversation among different ethnic groups in daily life. 'Piyazi' refers to a concrete object rather than an abstract idea, and thus it can be understood with much less effort.

Secondly, Uyghur speakers use their native language as a marker of their ethnic identity. As non-native Mandarin speakers, they unconsciously bring some of their native words into Mandarin when talking to speakers of Han Chinese, which is very common during language shifting. Native speakers of Han Chinese strive to understand the words in order not to embarrass the Uyghur speakers (Li 2002).

Thirdly, the lexical borrowings serve as emotional "ice breakers" between Han Chinese and Uyghur people. Lexical borrowings from another ethnic language can bridge the emotional distance, thus achieving better communicative performance (Wang 2006). Borrowing from or to another language is a way of showing friendliness and openness among different ethnic groups in Xinjiang.

Religious consideration is another reason for lexical borrowings. 'Zhurou' ('pork') has been changed to 'darou'. I failed to find academic data concerning the origin of the term 'darou'. This is not a core borrowing from Uyghur because 'darou' is a Chinese term, but it is commonly used by any ethnic group when referring to pork. Uyghurs in Xinjiang are primarily Muslims (Palmer et al. 2011) even though 'zhurou' is commonly used in the Han Chinese community. However, the term for pork has been changed to 'darou' because of this religious concern in Xinjiang. This change can be observed in other parts of China as well where there is a Muslim minority population.

In my view, there are two reasons that the use of 'darou' is preferred over 'zhourou'. First of all, the word 'zhu' (猪) is a curse word, it refers to someone who is stupid. Secondly, the speakers of Han Chinese in Xinjiang are aware of the taboo of eating pork in Muslim culture, and thus they subsequently adopt an alternative 
in order not to offend the Muslim population. The usage of 'zhurou' is not banned by law, yet the Han population have adopted the non-offensive form for pork.

\subsection{Words of Russian origin}

Uyghur borrowings account for the major borrowings into Mandarin in Xinjiang. However, there are a few words which came from other languages (for example, Russian and Persian). Lexical borrowings of Russian origin are much fewer than those of Uyghur origin due to Han Chinese and Uyghurs making up the majority of the population in Xinjiang. However, there are Russian borrowings in Mandarin. 'Moheyan' ('rolled tobacco') is derived from 'Махо́рка'. It maintains the phonetic features in the first two characters, 'mohe', yet it uses semantic loans in 'yan', which means 'tobacco'. 'Moheyan' is a popular rolled-up tobacco in Xinjiang, which originated between the $18^{\text {th }}$ and $19^{\text {th }}$ centuries. The tobacco was introduced to Xinjiang through a neighbouring country, Kazakhstan. It was first planted in Huo city and later expanded all over Xinjiang (Jiang et al. 1986). It can be inferred that Han Chinese borrowed this word from Uyghurs, who themselves borrowed it from Russian.

\subsection{Hybrid borrowings}

'Moheyan' can be identified as a hybrid borrowing because it applies both phonetic and semantic borrowing strategies. Another example, 'hainahua', is of Arabic origin 'حانَّ' ('henna') and is widely used by women in Xinjiang to dye their fingernails. 'Haina' is the phonetic borrowing part, together with 'hua' as a semantic feature, which means 'flower'. Thus, in both cases, 'yan' and 'hua' are added to the foreign word for semantic purposes.

The same hybrid borrowing can be found in 'Kanerjing' (坎儿井). It is a sloping underground irrigation system developed by Persians in ancient Iran in the first millennium BC (Wilson 2008). It was brought to China 2000 years ago, and now the length of the tunnel is close to 3,000 kilometres, connecting all the tunnels in Xinjiang (Huang-Kan 1990). The word 'kanerjing' is a hybrid borrowing form of 'qanat'. 'Qanat' has been adapted to 'kaner' phonetically, and 'jing' means well, which supplements the phonetic parts.

Some hybrid words are problematic to categorize. It is the case of words such as 'hulimatang' and 'miximixi'. They are created based on perceptive features from the non-native speaker's point of view. 'Hulimatang' (糊里麻唐) is a term commonly used by speakers of Han Chinese and Uyghurs; it refers to messy, unorganized behaviour. However, Uyghurs consider this word to be of Mandarin origin, yet Han Chinese speakers think of it is an Uyghur word (Xu 2012). Based on Miao's (2005) approach to categorizing lexical borrowings in Chinese, 'hulimatang' can be 
considered a hybrid word. However, it is not easy to decide whether it is a phonetic + semantic borrowing or not because the phonetic part sounds similar to Uyghur, but it is more of a made-up phonetic structure that sounds like Uyghur. Moreover, the semantic parts are not typical in the Mandarin expression either.

Guo (1999) observed similar cases of hybrid words of Chinese and Japanese origin. For example, 'miximixi' ('greetings') often appears in popular television and books from Japan. Chinese speakers consider this a Japanese word; however, Japanese speakers think this is a Chinese word. 'Miximixi' is different from 'hulimatang' because 'miximixi' is only used in television and books. There is no direct language contact in the case of 'miximixi', whereas in 'hulimangtang' there is direct language contact among Han Chinese and Uyghurs. Nevertheless, in these two examples, the way non-native speakers perceive other languages contributes to the creation of hybrid words. Even though the words do not make sense in their original language, the target language provides contextual meaning to these hybrids.

\section{Limitations}

The coexistence of different cultures and languages in Xinjiang have changed Mandarin in many aspects. The ethnic influence on Mandarin is not reflected solely by lexical borrowings. This paper only manages to present one aspect of the changes in Mandarin, and the examples are not exhaustively presented in this paper. The phonetic and sentence structures of Mandarin in Xinjiang have developed a few distinct features compared to standard Mandarin (Baki 2012). Future research in these areas could provide more insights about the language and cultures in Xinjiang.

\section{Conclusions}

This paper focuses on the influence of ethnic languages on Mandarin, which has not attracted much attention. The lexical borrowings from ethnic languages remind us of the language and cultural exchange among Han Chinese and other ethnic groups.

As we can see from the lexical borrowings, Chinese language and culture are tolerant to other languages. Language is a way to mark one's identity, but it is also a way to show friendliness. The emotional function of lexical borrowings from Uyghur to Mandarin should be highlighted. Speakers of Han Chinese choose to use terms in Uyghur as a way to break the ice and build friendships with Uyghurs. When Han Chinese speakers use terms borrowed from another language, it bridges the emotional distance even though Chinese and Uyghurs 
have different cultures, languages, and history. This openness will enable people to understand the differences among various ethnic groups.

\section{References}

Baki, Ablimit. 2012. Language Contact between Uyghur and Chinese in Xinjiang, PRC: Uyghur Elements in Xinjiang Putonghua. 41-62. https://www.degruyter. com/view/j/ijsl.2012.2012.issue-215/ijsl-2012-0028/ijsl-2012-0028.xml. (Last accessed: 21 April 2018).

Guo, Xi. 1999. 中国社会语言学 [Sociolinguistics in Chinese]. Nanjing: 南京大学 出版社.

Haspelmath, Martin. 2009. Lexical borrowing: Concepts and issues. Loanwords in the World's Languages: A Comparative Handbook: 35-54. https://www. researchgate.net/profile/Martin_Haspelmath/publication/279973916_Lexical_ borrowing_concepts_and_issues/links/5655dd7308ae1ef9297950d9/Lexical borrowing-concepts-and-issues.pdf. (Last accessed: 28 April 2018).

Huang, Wenfang-Kan, Yaoping. 1990. 新疆坎儿井的历史, 现状和今后发展 [The history, current status, and future development of qanat in Xinjiang]. 干旱区地 理 3: 33-37.

Jiang, Yuen-Tong, Daoru-Li, Yijun. 1986. 郭疆霍城莫合烟 [Rolled tobacco in Huo city, Xinjiang]. 中国烟草 3: 9 .

Li, Xiangrui. 2002. 对新疆汉语教学的几点思考 [Some thoughts on Chinese teaching in Xinjiang]. 语言与翻译 1: 57-59.

Lu, Wenli. 2011. 新疆汉语方言词汇浅论 [Discussion on Mandarin dialects in Xinjiang]. 语文学刊: 基础教育版 11: 17-19.

Miao, Ruiqin. 2005. Loanword Adaptation in Mandarin Chinese: Perceptual, Phonological and Sociolinguistic Factors. https://rucore.libraries.rutgers.edu/ rutgers-lib/38301/. (Last accessed: 18 April 2018).

Palmer, David-Shive, Glenn-Wickeri, Philip. 2011. Chinese Religious Life, 6162. New York: Oxford University Press.

Tsvetkov Y.-Dyer C. 2016. Cross-lingual bridges with models of lexical borrowing. Journal of Artificial Intelligence Research 55: 63-93.

Wang, Hairong. 2006. 探讨新疆汉话中的维吾尔语借词 [Discussion on lexical borrowing from Uyghur in Mandarin]. 和田师范专科学校学报 3: 157.

Wilson, Andrew. 2008. Hydraulic engineering and water supply. In John Peter Oleson (ed.), The Oxford Handbook of Engineering and Technology in the Classical World, 290-293. New York: Oxford University Press.

$\mathrm{Xu}$, Duohui. 2012. 新疆话糊里麻唐 (xulimataๆ) 等词语变异考 [Some thoughts on the lexical change of xulimataๆ]. 语言与翻译 2: 53-56. 